REVIEW

\title{
Raltegravir: first in class HIV integrase inhibitor
}

\author{
Zelalem Temesgen' \\ Dawd S Siraj \\ 'Mayo Clinic, Rochester, MN, USA; \\ ${ }^{2}$ East Carolina University Greenville, \\ NC, USA
}

\begin{abstract}
On October 16, 2007, the US Food and Drug Administration (FDA) approved raltegravir for treatment of human immunodeficiency virus (HIV)-1 infection in combination with other antiretroviral agents in treatment-experienced adult patients who have evidence of viral replication and HIV-1 strains resistant to multiple antiretroviral agents. Raltegravir is first in a novel class of antiretroviral drugs known as integrase inhibitors. It has demonstrated potent anti HIV activity in both antiretroviral treatment-naïve and experienced patients. The most common adverse events reported with raltegravir during phase 2 and 3 clinical trials were diarrhea, nausea, and headache. Laboratory abnormalities include mild elevations in liver transaminases and creatine phosphokinase.
\end{abstract}

Keywords: raltegravir, HIV, antiretroviral agents, integrase inhibitors

\section{Introduction}

A paradigm shift has occurred in the management of heavily treatment-experienced patients as a result of the introduction of new and potent antiretroviral drugs and our improved understanding of how best to incorporate these agents into a new treatment regimen. Thus, virological suppression has become an attainable goal for many of these patients. However, tolerability, adherence, and broad cross resistance within each class of antiretroviral drugs remain an issue and continue to limit the efficacy of current treatment options. Therefore, there is a continued need for new classes of drugs.

The integrase inhibitors target a viral enzyme that catalyses an essential process in the replication cycle of HIV - the insertion of HIV-1 proviral DNA into the host's cellular genome. Thus, they represent one of the most promising targets in the HIV life-cycle for therapeutic intervention. Raltegravir is a new, first-in-class drug in the class of integrase inhibitors and has demonstrated impressive potency in both treatmentnaïve and treatment experienced patients. This review summarizes available data on the clinical safety and efficacy of raltegravir as well as the general characteristics of the drug. Data for this review were obtained from papers published in the English language identified by searches of Medline, Current Contents, and references from relevant articles. The search terms used were "raltegravir", "integrase inhibitors", and "MK-0518". The review also includes data presented at major HIV-related meetings including Conference on Retroviruses and Opportunistic Infections, International AIDS Society Conference on HIV Pathogenesis and Treatment, Infectious Diseases Society of America annual meeting, International Congress on Drug Therapy in HIV Infection, Interscience Conference on Antimicrobial Agents and Chemotherapy, and International AIDS Conference. Data were also obtained from the Antiviral drugs Advisory Committee briefing Document, submitted by the manufacturer to the FDA.

\section{Structure and mechanism of action}

The integration of HIV-1 proviral DNA into the host cell genome is an obligate replication step of the HIV life cycle and occurs in three steps (LaFemina et al 1992; Hazuda et al 2000; Craigie et al 2001). Following reverse transcription of the viral RNA into a 
double-stranded DNA copy, the viral DNA remains associated with a "preintegration complex" that contains both viral and cellular proteins including the viral integrase protein. The integration of the viral DNA into the host chromosome is subsequently achieved through a series of DNA cutting and joining reactions. First, two nucleotides are removed from each 3'-end of the viral DNA, a process termed 3'-end processing. In the second step, termed DNA strand transfer, the processed viral DNA ends are inserted or joined into the host DNA. The HIV-1 integrase catalyses these first two steps of integration. In the third step, cellular enzymes repair the single gaps in the DNA chain by removing the two unpaired nucleotides at the 5 '-ends of the viral DNA.

The development of raltegravir evolved from the discovery of specific compounds that contained a distinct $\beta$ diketo acid (DKA) moiety with potent and selective inhibitory activity against the strand transfer step of integration (Hazuda et al 1999, 2005; Espeseth et al 2000; Pais et al 2002; Embrey et al 2005). Substitution of the 1,3-DKA moiety by 8 -hydroxy- $(1,6)$ naphthyridine led to the synthesis of more metabolically stable candidates represented by the naphthyridine carboxamide compounds. Further modifications of these compounds led to the synthesis of a new naphthyridine derivative, raltegravir (formerly known as L-900612 and MK-0518) and its emergence as a candidate for further development. The full chemical name of raltegravir is 4-[N-(4-Fluorobenzyl)carbamoyl]-1-methyl-2-[1-methyl1-(5-methyl-1,3,4-oxadiazol-2-ylcarboxamido)ethyl]-6-oxo1,6-dihydropyrimidin-5-olate.

\section{Pharmacology and metabolism}

Raltegravir is rapidly absorbed with a bioavailability of at least $32 \%$. With twice daily dosing, pharmacokinetic steady state is achieved within approximately the first 2 days of dosing (Isentress 2007; Isentress FDA Briefing Document 2007; Kassahun et al 2006). Raltegravir is approximately $83 \%$ bound to human plasma proteins. The apparent terminal $t \frac{1}{2}$ of raltegravir in humans is approximately 9 hours with a shorter $\alpha$-phase half-life ( $\sim$ 1hour) accounting for much of the AUC. This and its elimination profile support the use of a twice-daily dosing regimen for raltegravir. High fat meal slows the rate of absorption of raltegravir but also increases its exposure (area under the curve, AUC). Following a single $400 \mathrm{mg}$ dose administration of raltegravir with high fat meal, the time to reaching maximum plasma concentration $\left(\mathrm{C}_{\max }\right)$ was delayed by 7.5 hours and the $\mathrm{C}_{\max }$ itself was decreased by $34 \%$. However, the AUC was increased by approximately 19\%. Therefore, raltegravir may be administered without regard to food. The major mechanism of clearance of raltegravir in human beings is metabolism via UGT1A1-mediated glucuronidation (Isentress 2007; Isentress FDA Briefing Document 2007; Kassahun et al 2006, 2007). After an oral administration of radiolabeled raltegravir, approximately 51\% was excreted in feces while 31\% was excreted in urine. No adjustment of dosing is necessary for age, gender, body mass index, HIV infection status, hepatic and renal function (Isentress 2007; Isentress FDA Briefing Document 2007).

\section{Interactions}

Raltegravir is metabolized by glucuronidation. At a concentration up to $100 \mu \mathrm{M}$, raltegravir does not inhibit or induce nor is it a substrate of cytochrome P450 enzymes. Thus, it is expected that interactions with drugs metabolized by the CYP450 system, including protease inhibitors and nonnucleoside reverse transcriptase inhibitors, are unlikely (Isentress FDA Briefing Document 2007).

Atazanavir is a known inhibitor of UGT1A1, the primary pathway of clearance of raltegravir, and was selected to assess the greatest potential for increases in raltegravir pharmacokinetics (Mistry et al 2007). Both atazanavir alone and in combination with ritonavir were investigated in phase 1 studies. Additional data on this interaction was also obtained from the phase 2 population $\mathrm{PK}$ data. In those studies, raltegravir plasma levels were increased when coadministered with atazanavir, consistent with inhibition of UGT1A1. The increases, however, were on the whole modest $(30 \%-70 \%$ increases in AUC) and not considered clinically meaningful. Additionally, concomitant use of raltegravir and atazanavir was well tolerated in the phase 2 and phase 3 studies. Based on these data, atazanavir may be coadministered with raltegravir without adjustment in the dose of raltegravir.

Tenofovir is known to significantly affect the pharmacokinetics of other antiretroviral agents through mechanisms that are not entirely clear. Phase 1 and 2 studies that include coadministration of raltegravir and tenofovir concluded that there is no clinically meaningful interaction and the two drugs can be coadministered without dose adjustment (Wenning et al 2006a). Similarly, the interaction of raltegravir with efavirenz, etravirine and tipranavir with low dose of ritonavir was studied and found not to be clinically meaningful (Iwamoto et al 2006a; Wenning et al 2006b). Rifampin is a potent inducer of cytochrome P450. Coadministration of rifampin with raltegravir decreased raltegravir 12-hour concentration by an average of $61 \%$ (Iwamoto et al 2006b). The drop in raltegravir level with potent cytochrome P450 inducers is most likely clinically significant and caution should be used if rifampin or similar potent CYP450 inducers 
such as phenytoin and phenobarbital are coadministered with raltegravir (Kassahun et al 2006).

Coadministration of oral contraceptives (Ortho TriCyclen or generic equivalent) with raltegravir for 21 days did not substantially alter plasma exposure levels of either ethinyl estradiol or norelgestromin in healthy female subjects (Anderson et al 2007).

\section{Antiviral activity In vitro studies}

Raltegravir demonstrated antiviral activity in cell culture against a broad panel of HIV isolates, including primary isolates from a variety of subtypes, isolates resistant to protease inhibitors (PIs), nucleoside reverse transcriptase inhibitors (NRTIs), and non-nucleoside reverse transcriptase inhibitors (NNRTIs), and even against simian immunodeficiency virus (SIV) (Miller et al 2006). It inhibited the strand transfer activity of purified HIV-1 integrase in vitro with an IC50 of 2-7 nM. Raltegravir was also $>1000$-fold more selective for HIV-1 integrase as compared with other phosphoryltransferases tested, including the polymerase and RNase $\mathrm{H}$ activities of HIV-1 reverse transcriptase and the human polymerases $\alpha, \beta$, and $\gamma$.

\section{Clinical studies}

\section{Phase 2 clinical trials}

\section{Study 004}

Study 004 was a multicenter, double-blind, randomized, placebo-controlled, 2-part, dose-ranging study of raltegravir in antiretroviral naïve HIV-infected adults with a plasma HIV-1 RNA level of 5000 copies/mL or greater and a CD4+ T-cell count of at least 100 cells $/ \mathrm{mm}^{3}$ at screening. The first part of the study was a 10-day monotherapy phase that compared 4 doses of raltegravir $(100,200,400$, and $600 \mathrm{mg})$ to placebo, each given twice daily, in 35 patients (Markowitz et al 2006). Patients were stratified at entry by initial plasma HIV-1 RNA level ( $<$ or $>50,000$ copies $/ \mathrm{mL}$ ). Baseline plasma HIV-1 RNA levels were comparable among all 5 groups with mean values ranging from 4.53 to $4.97 \log 10$ copies $/ \mathrm{mL}$. All 35 patients completed the 10-day study. The antiviral response was similar among all raltegravir groups (mean decreases in $\log 10$ HIV RNA level of 1.93, 1.98, 1.66, and 2.16 copies/

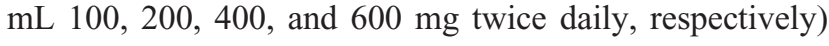
and was significantly greater compared with placebo (mean decreases in $\log 10$ HIV RNA -0.17; p $<0.001$ ). At least half of the patients in each raltegravir dose group achieved an HIV RNA level $<400$ copies/mL by day 10 . There were no differences in the slope of HIV RNA decline between patients with HIV RNA level $<$ or $>50,000$ copies $/ \mathrm{mL}$ at study entry. Drug-related clinical adverse events were reported in 10 patients ( 8 among the 4 raltegravir treatment groups and 2 patients in the placebo group). These were mild or moderate in intensity. There were no serious adverse events and no discontinuations because of adverse experiences. One patient in the 100-mg raltegravir group experienced a grade 1 increase in alanine transferase (ALT) on day 5 which resolved by day 10 without interruption of therapy.

The second part of study 004 enrolled 198 antiretroviral treatment-naive patients (including 30 patients from the first part of study 004) and compared the safety and efficacy of the 4 different doses of raltegravir described above to that of efavirenz, each drug combined with tenofovir and lamivudine (Markowitz et al 2007). Baseline characteristics were in general comparable across all study arms. Mean baseline HIV-1RNA values were 4.6-4.8 $\log 10$ copies/mL across all groups. Mean baseline CD4 cell counts ranged from 271 to 314 cells $/ \mathrm{mm}^{3}$. All participants had documented genotypic susceptibility to TDF, 3TC, and EFV. At 48 weeks, virologic efficacy was comparable between raltegravir and efavirenz with all 5 treatment arms demonstrating a greater than $2.2 \log _{10}$ decline in HIV-1 RNA. Virologic failure occurred at similar rates $(3 \%)$. Significantly more patients in the raltegravir arms achieved HIV-1 RNA $<50$ copies/mL at week 4 and 8 than patients in the EFV arm but these differences were no longer statistically significant by week 12 and responses remained similar to week 48. Mean increases from baseline in CD4+ T cell count exceeded 100 cells $/ \mathrm{mm}^{3}$ in all arms. Adverse events were uncommon, were mild to moderate in nature, and were generally similar across all treatment groups.

\section{Study 005}

Study 005 was a multicenter, double-blind, randomized, placebo-controlled, dose-ranging study that assessed the safety and efficacy of raltegravir when added to optimized background regimens in 178 antiretroviral experienced HIVinfected adults with a plasma HIV-1 RNA level above 5000 copies $/ \mathrm{mL}$ and a CD4+ T-cell count of at least 50 cells $/ \mathrm{mm}^{3}$ at screening (Grinsztejn et al 2007). Participants were also required to be on stable antiretroviral therapy for more than 3 months and have documented genotypic or phenotypic resistance to at least one non-nucleoside reverse transcriptase inhibitor, one nucleoside reverse transcriptase inhibitor, and one protease inhibitor. Each patient's optimized background regimen was selected by investigators prior to randomization based on results from all available genotypic and phenotypic 
resistance tests as well as the patient's antiretroviral treatment history. Patients were randomized in a $1: 1: 1: 1$ ratio to one of three doses of raltegravir (200 mg, $400 \mathrm{mg}$, or $600 \mathrm{mg}$ ) or placebo twice daily. At randomization, patients were also stratified by enfuvirtide use and by the degree of resistance to PIs at study entry. The primary endpoints were the change in HIV-1 RNA from baseline at week 24 and safety. The demographic and other baseline characteristics of the patients were comparable in all four groups. At 24 weeks, virologic and immunologic treatment outcomes were better for each raltegravir arm compared to the placebo arm ( $p<0.0001)$. Table 1. The treatment differences between raltegravir and placebo groups were consistent across all phenotypic and genotypic sensitivity scores (PSS and GSS). GSS and PSS refer to the number of drugs in the regimen to which the virus is deemed to be sensitive according to the baseline genotype or phenotype. For example, a genotypic sensitivity score of 0 means that participants received no other active drugs in addition to raltegravir or placebo). The use of enfuvirtide in the optimized background regimen was associated with improved outcomes with both raltegravir and placebo. Raltegravir at all doses had a comparable safety profile to that of placebo. There were no apparent dose-related toxicities.

\section{Phase 3 clinical trials}

On the basis of the data from study 004 and 005 the dose of $400 \mathrm{mg}$ twice daily of raltegravir was selected for phase III studies.

\section{BENCHMRK}

BENCHMRK-1 and -2 (study 018 and 019) are two parallel, identical, ongoing phase III double-blind, placebo-controlled studies, that are evaluating the efficacy and safety of raltegravir plus an optimized background regimen in patients with triple class resistant HIV-1 virus (Cooper et al 2007; Steigbigel et al 2007). BENCHMRK 1 was conducted in Europe, Asia and the Pacific, and Peru. BENCHMRK 2 was conducted in North, Central, and South America. Eligible participants were those failing antiretroviral therapy with triple antiretroviral class resistance and were randomized 2:1 to raltegravir $400 \mathrm{mg}$ twice daily or placebo. Participants were required to have plasma HIV-1 RNA level above 1000 copies/mL and documented genotypic or phenotypic resistance to at least one non-nucleoside reverse transcriptase inhibitor, one nucleoside reverse transcriptase inhibitor, and one protease inhibitor. An optimized background therapy (OBT) was selected by the treating physicians. Select investigational drugs (eg, darunavir) were permitted to be part of OBT. The demographic and other baseline characteristics of the patients were comparable between the raltegravir and placebo arms in each study (Table 2). The primary endpoint was the proportion of patients that achieved HIV-1 RNA $<400$ copies/mL at week 16 . Additional efficacy endpoints included percentage of patients with HIV RNA $<50$ copies/mL as well as change from baseline in HIV-1 RNA levels and CD4 cell counts. At 16 weeks, the raltegravir arm was found to be superior in its antiretroviral effect compared to the placebo arm (Table 3). Superior efficacy of the raltegravir arms over placebo was maintained regardless of baseline CD4 count, HIV-1 RNA values as well as genotypic and phenotypic scores in the OBT. Adverse events were similar between groups. 24-week combined results of the BENCHMRK studies confirm that the superior efficacy of the raltegravir arms was sustained (Kumar et al 2007) (Table 4). Analysis of these 24-week combined results also provided an insight into differences in treatment outcome based on the drugs used in the OBT (Table 5). While the raltegravir arms remained more effective than the placebo arms regardless of the composition of the OBT, differences in treatment outcome were smaller if darunavir and enfurvitide were used together in patients not previously exposed to them.

Table I Study 005: 24-week results

\begin{tabular}{|c|c|c|c|c|}
\hline & Raltegravir ${ }^{\mathrm{a}} 200 \mathrm{mg}$ & Raltegravir $^{\mathrm{a}} 400 \mathrm{mg}$ & Raltegravir $^{\mathrm{a}} 600 \mathrm{mg}$ & Placebo $^{a}$ \\
\hline$\underline{\mathbf{n}}$ & 43 & 45 & 45 & 45 \\
\hline $\begin{array}{l}\text { Change from baseline in HIV-I RNA } \\
\text { (log } 10 \text { copies } / \mathrm{mL} \text { ) }\end{array}$ & -1.80 & -1.87 & -1.84 & -0.35 \\
\hline $\begin{array}{l}\text { Proportion of patients with HIV-I RNA } \\
<400 \text { Copies } / \mathrm{mL}\end{array}$ & $69.8 \%$ & $71.1 \%$ & $71.1 \%$ & $15.6 \%$ \\
\hline $\begin{array}{l}\text { Proportion of patients with HIV-I RNA } \\
<50 \text { copies } / \mathrm{mL}\end{array}$ & $65.1 \%$ & $55.6 \%$ & $66.7 \%$ & $13.3 \%$ \\
\hline $\begin{array}{l}\text { Change from baseline in CD4 cell count } \\
\text { (cells } / \mu \mathrm{L} \text { ) }\end{array}$ & 62.9 & 112.8 & 94.1 & 5.4 \\
\hline
\end{tabular}

aPlus optimized background regimen. 
Table 2 Selected baseline characteristics BENCHMRK-I and -2 trials

\begin{tabular}{|c|c|c|c|c|}
\hline \multirow[b]{3}{*}{$\mathbf{n}$} & \multicolumn{2}{|c|}{ BENCHMRK-I } & \multicolumn{2}{|c|}{ BENCHMRK-2 } \\
\hline & Raltegravir & Placebo & Raltegravir & Placebo \\
\hline & 232 & 118 & 230 & 119 \\
\hline Age & 46.1 & 43.7 & 45.3 & 46.5 \\
\hline Male & $84.1 \%$ & $87.3 \%$ & $91.3 \%$ & $89.9 \%$ \\
\hline Baseline CD4 count (mean) & 156.4 & 152.8 & 146.4 & 163.2 \\
\hline $\begin{array}{l}\text { Baseline HIV-I RNA (mean) } \\
\log 10 \text { copies } / \mathrm{mL}\end{array}$ & 4.6 & 4.5 & 4.7 & 4.7 \\
\hline AIDS diagnosis & $93.5 \%$ & $89.8 \%$ & $90.9 \%$ & $92.4 \%$ \\
\hline $\begin{array}{l}\text { Prior antiretroviral use in } \\
\text { years (median) }\end{array}$ & 10.6 & 10.3 & 9.6 & 10.1 \\
\hline $\begin{array}{l}\text { Number of antiretrovirals } \\
\text { used in the past }\end{array}$ & 12 & 12 & 12 & 12 \\
\hline $\begin{array}{l}\text { Enfurvitide }^{\mathrm{a}} \text { in optimized } \\
\text { background regimen }\end{array}$ & $37.9 \%$ & $36.4 \%$ & $37.8 \%$ & $38.7 \%$ \\
\hline Resistance to $\geq \mathrm{Pls}$ & $97 \%$ & $94.9 \%$ & $96.5 \%$ & $95.8 \%$ \\
\hline
\end{tabular}

Derived from Isentress FDA Briefing Document (2007).

ancludes those already enfuvirtide experienced.

Abbreviation: Pls, protease inhibitors.

\section{Safety}

Raltegravir safety data are obtained primarily from the phase 2 and 3 studies of raltegravir described above - study 004, 005, and the BENCHMRK studies. Study 004 in particular is an important source of safety information on raltegravir since participants in both arms of the study received the exact same regimen (tenofovir plus lamivudine) with the exception of the two drugs that were being compared, raltegravir and efavirenz.

In study 004, the most frequently reported clinical adverse events in the raltegravir groups (all doses combined) regardless of causality were: diarrhea $(18.8 \%)$, nausea $(18.1 \%)$,

Table 3 BENCHMRK-I and 2: treatment outcome at week 16

\begin{tabular}{|c|c|c|c|c|}
\hline \multirow[b]{3}{*}{$\underline{\mathbf{n}}$} & \multicolumn{2}{|c|}{ BENCHMRK-I } & \multicolumn{2}{|c|}{ BENCHMRK-2 } \\
\hline & Raltegravir & Placebo & Raltegravir & Placebo \\
\hline & 232 & 118 & 230 & 119 \\
\hline $\begin{array}{l}\text { Proportion of patients with } \\
\text { HIV-I RNA }<400 \text { copies } / \mathrm{mL}\end{array}$ & $76.7 \%$ & $40.7 \%$ & $77 \%$ & $42.9 \%$ \\
\hline $\begin{array}{l}\text { Proportion of patients with } \\
\text { HIV-I RNA < } 50 \text { copies } / \mathrm{mL}\end{array}$ & $60.8 \%$ & $33.1 \%$ & $61.7 \%$ & $36.1 \%$ \\
\hline $\begin{array}{l}\text { Proportion of patients with }>\text { I } \\
\text { log decline in HIV-I RNA }\end{array}$ & $84.9 \%$ & $41.5 \%$ & $82.6 \%$ & $50.4 \%$ \\
\hline $\begin{array}{l}\text { Mean change in HIV-I RNA } \\
\text { (log } 10 \text { copies } / \mathrm{mL} \text { ) from } \\
\text { baseline }\end{array}$ & -1.85 & -0.78 & -1.92 & -1.06 \\
\hline $\begin{array}{l}\text { Mean change in CD4 count } \\
\left(\text { cells } / \mathrm{mm}^{3}\right) \text { from baseline }\end{array}$ & +82.7 & +31.3 & +85.1 & +39.7 \\
\hline $\begin{array}{l}\text { Virologic failure - non } \\
\text { responder }^{\mathrm{a}}\end{array}$ & $1.7 \%$ & $37.3 \%$ & $3.9 \%$ & $28.6 \%$ \\
\hline Virologic failure - rebound ${ }^{b}$ & $12.1 \%$ & $16.1 \%$ & $12.6 \%$ & $19.3 \%$ \\
\hline
\end{tabular}


Table 4 Combined BENCHMRK I and 2, 24-week treatment outcome

\begin{tabular}{lll}
\hline & BENCHMRK I and 2 & Placebo \\
\cline { 2 - 3 } $\mathbf{n}$ & $\mathbf{4 4 8}$ & $\mathbf{2 3 0}$ \\
\hline $\begin{array}{l}\text { Proportion of patients with } \\
\text { HIV-I RNA }<50 \text { copies } / \mathrm{mL}\end{array}$ & $65 \%$ & $35 \%$ \\
$\begin{array}{l}\text { Mean change in CD4 count } \\
\left(\text { cells } / \mathrm{mm}^{3} \text { ) from baseline }\right.\end{array}$ & +84 & +37 \\
\hline
\end{tabular}

upper respiratory infection (16.9\%), headache $(16.3 \%)$, dizziness $(11.9 \%)$, nasopharyngitis $(10.6 \%)$, and insomnia $(10.6 \%)$. The most frequently reported (incidence $>10 \%$ ) drug-related clinical adverse experience in the raltegravir treatment groups (all doses combined) was nausea (11.3\%). There was no apparent association between the frequency of clinical adverse events (drug-related or not) and increasing raltegravir dose. Elevations of alanine aminotransferase (ALT) or aspartate aminotransferase (AST) were reported in $3.8 \%$ of patients in the raltegravir treatment groups (all doses combined) compared to $5.3 \%$ of patients in the efavirenz group. Of note, raltegravir, in contrast to efavirenz, had no appreciable effect on total cholesterol, LDL cholesterol, or triglycerides (Kassahun et al 2007; Markowitz et al 2007).

Understanding safety data from studies in treatmentexperienced patients can be a challenge because of the additional confounding factors including differences in the optimized background regimens and advanced stage of HIV disease. In study 005 as well as the 2 BENCHMRK studies, adverse events were generally balanced between the raltegravir arms and the placebo arms (Cooper et al 2007; Grinsztejn et al 2007; Kassahun et al 2007; Steigbigel et al 2007). Serious adverse events were reported in $10.7 \%$ of raltegravir recipients versus $12.8 \%$ of patients receiving placebo. The most common drug-related clinical adverse events were diarrhea (3.7\% vs 3.5\%), nausea ( $2.2 \%$ vs $3.2 \%)$, and headache $(2.2 \%$ vs $1.4 \%)$. The proportions of laboratory adverse events were comparable between the raltegravir and the placebo group. However, three laboratory adverse events were reported more frequently in patients in the raltegravir groups compared to patients in the placebo groups: elevations of ALT (4.5\% vs 2.1\%); elevations of AST (4.3\% vs $2.5 \%)$; elevations of creatine phosphokinase (CPK) $(3.2 \%$ vs $0.7 \%$ ). As regards the aminotransferase elevations, they were usually transient and did not generally lead to study or drug discontinuation. In patients with hepatitis B and/or C coinfection, increases in ALT and AST did not differ between raltegravir and placebo groups: $5.1 \%$ and $7.7 \%$, respectively, for the raltegravir group and $5.1 \%$ and $5.1 \%$, respectively, for the placebo group. As regards the elevations of creatine phosphokinase, many of these elevations were transient and some were thought to be related to physical exercise. No patient discontinued therapy due to increased CPK.

Nine deaths were reported in the BENCHMRK studies: 6 from the raltegravir group $(1.2 \%)$ and 3 from the placebo group (1.1\%). In general, these deaths were related to severe opportunistic infection and/or malignancy. None of these deaths has been determined to be drug-related (Kassahun et al 2007).

Malignancies were reported at a higher rate in patients on raltegravir in the phase 2 and 3 studies compared to patients on a comparator arm: $19(2.5 \%)$ vs $5(1.5 \%)$. However, the difference between the two groups was not sustained when the rates of malignancies were adjusted for the patient years of exposure to the study drug (Kassahun et al 2007). Thus, the patient-year adjusted rates of malignancies per 100 patient-years were 2.32 in the raltegravir arms and 1.92 in the comparator groups with a relative risk of 1.209 (95\% confidence interval $0.44,4.14)$. The malignancies reported include Kaposi's sarcoma, nonHodgkin's lymphoma, anogenital squamous cell cancer, rectal cancer, hepatocellular cancer, and non-melanoma skin cancer. Eight of the 19 cancers in the raltegravir were recurrent; the remaining 11 occurred within $\leq 3$ months of enrollment. The median CD4 cell count of the patients receiving raltegravir who ultimately developed a malignancy was 122 cells $/ \mathrm{mm}^{3}$. All patients with malignancy also had a history of AIDS.

\section{Resistance}

Current knowledge regarding HIV resistance to raltegravir is incomplete and evolving. In vitro studies have identified

Table 5 Combined BENCHMRK I and 2,24-week results - response according to drugs in optimized background therapy

\begin{tabular}{lll}
\hline & $\begin{array}{l}\text { BENCHMRK } \\
\text { I and 2 }\end{array}$ & Placebo \\
\cline { 2 - 3 } $\mathbf{n}$ & $\mathbf{4 4 8}$ & $\mathbf{2 3 0}$ \\
\hline $\begin{array}{l}\text { Proportion of patients with HIV-I } \\
\text { RNA }<50 \text { copies/mL }\end{array}$ & $65 \%$ & $35 \%$ \\
$\begin{array}{l}\text { Proportion of patients with first } \\
\text { time darunavir and enfurvitide use } \\
\text { with HIV-I RNA }<50 \text { copies/mL }\end{array}$ & $80 \%$ & $74 \%$ \\
$\begin{array}{l}\text { Proportion of patients with first time } \\
\text { darunavir but no enfurvitide use with }\end{array}$ & $68 \%$ & \\
$\begin{array}{l}\text { HIV-I RNA }<50 \text { copies/mL } \\
\text { Proportion of patients with first time } \\
\text { enfuvirtide but no darunavir use with } \\
\text { HIV-I RNA }<50 \text { copies/mL }\end{array}$ & $82 \%$ & $48 \%$ \\
\hline
\end{tabular}


a series of specific amino acid changes in the gene coding for the integrase enzyme that occur over time during culture in increasing raltegravir concentrations. The first change observed was Q148K followed by E138A and G140A with acquisition of additional mutations with higher drug concentrations. Introduction of these mutations (Q148K, E138A/ Q148K, and E138A/G140A/Q148K) into a wild-type virus resulted in a substantial fold-shift (46-508 fold) in raltegravir IC50. An additional effect of these mutations was a reduction in viral replication (Kassahun et al 2007).

Studies of treatment-emergent resistance to raltegravir in the phase 2 and 3 clinical trials have provided further insight (Hazuda et al 2007; Kassahun et al 2007). Most patients experiencing virologic failure while receiving a raltegravir-containing regimen will fail with viruses that display integrase mutations that confer resistance to raltegravir (in particular if they are treatment-experienced patients). It appears that there are two distinct pathways of resistance one involving amino acid residue 148 (Q148H, Q148K, or Q148R) the other involving N155H. These mutations confer 10- to 25-fold, respectively, decreased susceptibility to raltegravir. Additional secondary mutations such as L74M, E92Q, T97A, E138K, G140S, V151I, G163G/R, and D232D/N may also be present in raltegravir virologic failures. These secondary mutations by themselves contribute only in a modest way to resistance to raltegravir. However, when combined with either of the two primary mutations, they lead to substantial further decreases in raltegravir susceptibility. Further analysis of virologic failure samples from the 005 study as well as from a phase II trial of elvitegravir, another integrase inhibitor, has indicated that there may be substantial cross resistance between these two drugs (Hazuda et al 2007; $\mathrm{McColl}$ et al 2007). A recent case report appears to corroborate these findings (DeJesus et al 2007). Two patients participating in the phase 2 elvitegravir phase II GS-US-183-0105 trial were allowed to switch from elvitegravir/ritonavir to raltegravir with the same background regimen for one week after experiencing virologic failure. One patient had A91Q, Q95Q/K, T97T/A, N155H at virologic failure. The second had H51Y, E138E/K, P145P/S, S147G, Q148R at virologic failure. No significant reductions in HIV-1 RNA for either patient were noted after 1 week of raltegravir substitution. These results led to the termination of the pilot study under which this evaluation was conducted.

\section{Conclusion}

Raltegravir, a novel integrase inhibitor has demonstrated impressive antiviral potency in heavily treatment-experienced patients as well as in patients naïve to antiretroviral therapy. These impressive results render raltegravir a viable option for treating patients of varying treatment experience but the optimal role of raltegravir in the sequencing of antiretroviral therapy, and the optimal antiretroviral agents to combine it with remain to be defined. The availability of raltegravir, together with other recently introduced new antiretroviral drugs, has the potential to alter the prevailing treatment paradigm of combining two NRTIs with a PI or an NNRTI as the corner stone of highly active antiretroviral therapy.

\section{Disclosures}

Neither author is employed by manufactures of antiretroviral drugs. Furthermore, no external party has directed the content or participated in the writing of this review.

\section{References}

Anderson MS, Wenning L, Moreau A, et al. 2007. Effect of raltegravir on the pharmacokinetics of oral contraceptives. $47 \mathrm{th}$ Interscience Conference on Antimicrobial Agents and Chemotherapy, Chicago, IL. September 17-20, 2007. Abstract A-1425.

Cooper D, Gatell J, Rockstroh J, et al. 2007. Results of BENCHMRK-1, a phase III study evaluating the efficacy and safety of MK-0518, a novel HIV-1 integrase inhibitor, in patients with triple-class resistant virus. Program and abstracts of the 14th Conference on Retroviruses and Opportunistic Infections; February 25-28, 2007; Los Angeles, California. Abstracts 105aLB.

Craigie R. 2001. HIV integrase, a brief overview from chemistry to therapeutics. J Biol Chem, 276:23213-6.

DeJesus E, Cohen C, Elion R, et al. 2007. First report of raltegravir (RAL, MK-0518) use after virologic rebound on elvitegravir (EVT, GS 9137). Program and abstracts of the 4th International AIDS Society Conference on HIV Pathogenesis, Treatment and Prevention; July 22-25, 2007; Sydney, Australia. Abstract TUPEB032.

Embrey MW, Wai JS, Funk TW, et al. 2005. A series of 5-(5,6)dihydrouracil substituted 8-hydroxy-[1,6]naphthyridine-7-carboxylic acid 4-fluorobenzylamide inhibitors of HIV-1 integrase and viral replication in cells. Bioorg Med Chem Lett, 15:4550-4.

Espeseth AS, Felock P, Wolfe A, et al. 2000. HIV-1 integrase inhibitors that compete with the target DNA substrate define a unique strand transfer conformation for integrase. Proc Natl Acad Sci USA, 97:11244-9.

Grinsztejn B, Nguyen B, Katlama C, et al. 2007. Safety and efficacy of the HIV-1 integrase inhibitor raltegravir (MK-0518) in treatment-experienced patients with multidrug-resistant virus: a phase II randomised controlled trial. Lancet, 369:1261-9.

Hazuda D, Blau CU, Felock P, et al. 1999. Isolation and characterization of novel human immunodeficiency virus integrase inhibitors from fungal metabolites. Antivir Chem Chemother, 10:63-70.

Hazuda DJ, Anthony NJ, Gomez RP, et al. 2004. A naphthyridine carboxamide provides evidence for discordant resistance between mechanistically identical inhibitors of HIV-1 integrase. Proc Natl Acad Sci USA, 101:11233-8.

Hazuda DJ, Felock P, Witmer M, et al. 2000. Inhibitors of strand transfer that prevent integration and inhibit HIV-1 replication in cells. Science, 287:646-50.

Hazuda DJ, Miller MD, Nguyen BY, et al. 2007. Resistance to the HIVintegrase inhibitor raltegravir: analysis of protocol 005, a phase II study in patients with triple-class-resistant HIV-1 infection. Program and abstracts of the 16th International HIV Drug Resistance Workshop; June 12-16, 2007; Barbados, West Indies. Abstract 8. 
Isentress prescribing information. Merck and Co., Iin., Whitehouse Station, NJ 08889, USA.

ISENTRESSTM (raltegravir) $400 \mathrm{mg}$ for treatment of HIV (NDA 22-145) 2007. FDA Briefing Document. Accessed October 15, 2007.URL: http://www. fda.gov/OHRMS/DOCKETS/AC/07/briefing/2007-4314b1-01-Merck. pdf.

Iwamoto M, Wenning LA, Liou SY, et al. 2006. Rifampin (RIF) modestly reduces plasma levels of MK-0518. 8th international congress on drug therapy in HIV infection, Glasgow. Nov 2006. Abstract 20061; p299.

Iwamoto M, Wenning LA, Petry AS et al. 2006. Minimal effect of ritonavir (RTV) and efavirenz (EFV) on the pharmacokinetics (PK) ofMK-0518. 46th Annual ICAAC Meeting. San Francisco, CA, 2006: Abstract 2006b; A-373.

Kassahun K, McIntosh I, Cui D, et al. 2007. Metabolism and disposition in humans of raltegravir (MK-0518), an anti-AIDS drug targeting the HIV-1 integrase enzyme. Drug Metab Dispos, 35:1657-63.

Kassahun K, McIntosh I, Hreniuk D, et al. 2006. Absorption, metabolism and excretion of MK-0518, a potent HIV-1 integrase inhibitor, in healthy male volunteers. 46th Annual Interscience Conference on Antimicrobial Agents and Chemotherapy, San Francisco, California, USA, Sept 27-30, 2006: abstract A-372.

Kumar P, Cooper D, Steigbigel R, et al. 2007. Efficacy of raltegravir, an HIV integrase inhibitor, in combination with regimens containing enfuvirtide, darunavir, or tipranavir in patients with triple-class resistant virus: combined results from Benchmrk-1 and Benchmrk-2. European AIDS Conference. October 24-27, 2007. Madrid. Abstract P7.2/06.

LaFemina RL, Schneider CL, Robbins HO, et al. 1992. Requirement of active human immunodeficiency virus type 1 integrase enzyme for productive infection of human T-lymphoid cells. J Virol, 66:7414-19.

Markowitz M, Morales-Ramirez JO, Nguyen BY, et al. 2006. Antiretroviral activity, pharmacokinetics, and tolerability of MK-0518, a novel inhibitor of HIV-1 integrase, dosed as monotherapy for 10 days in treatment-naive HIV-1-infected individuals. J Acquir Immune Defic Syndr, 43:509-15.
Markowitz M, Nguyen BY, Gotuzzo E, et al. 2007. Rapid and durable antiretroviral effect of the HIV-1 integrase inhibitor raltegravir as part of combination therapy in treatment-naive patients with HIV-1 infection: results of a 48-week controlled study. J Acquir Immune Defic Syndr, 46:125-33.

McColl DJ, Fransen S, Gupta S, et al. 2007. Resistance and cross-resistance to first-generation integrase inhibitors: insights from a phase II study of elvitegravir (GS-9137). Program and abstracts of the 16th International HIV Drug Resistance Workshop; June 12-16, 2007; Barbados, West Indies. Abstract 9.

Miller MD, Witmer MV, Stillmock KA, et al. 2006. Biochemical and antiviral activity of MK-0518, a potent HIV integrase inhibitor. In: program and abstracts of XVI International AIDS Conference; 2006, Toronto.

Mistry G, Wenning L, Petry A, et al. 2007. Atazanavir modestly increases plasma levels of MK-0518. 4h International AIDS Society Conference on HIV Pathogenesis, Treatment and Prevention. Sidney, Australia, 2007. Abstract number: 1985.

Pais GCG, Zhang XC, Marchand C, et al. 2002. Structure activity of 3-aryl1,3-diketo-containing compounds as HIV-1 integrase inhibitors. J Med Chem, 45:3184-94.

Steigbigel R, Kumar P, Eron J, et al. 2007. Results of BENCHMRK-2, a phase III study evaluating the efficacy and safety of MK-0518, a novel HIV-1 integrase inhibitor, in patients with triple-class resistant virus. Program and abstracts of the 14th Conference on Retroviruses and Opportunistic Infections; February 25-28, 2007; Los Angeles, California. Abstracts 105bLB.

Wenning LA, Friedman E, Kost JT, et al. 2006. Lack of significant drug interaction between MK-0518 and tenofovir disoproxil fumarate. 46th Annual ICAAC Meeting. San Francisco, CA, 2006: Abstract 2006b; A-375.

Wenning LA, Hanley H, Stone J, et al. 2006. Effect of tipranavir + ritonavir on pharmacokinetics of MK-0518. 46th Annual ICAAC Meeting. San Francisco, CA, 2006: Abstract 2006a; A-374. 